\title{
BEAM BASED ALIGNMENT OF BPM AND ORBIT STABILIZATION AT THE PHOTON FACTORY STORAGE RING
}

\author{
T. Honda, T. Obina, T. Miyajima and Y. Kobayashi, KEK, Tsukuba, Japan
}

\begin{abstract}
Beam orbit of the PF ring is adjusted to deliver the photon beams on the proper axes of all experimental beam lines. During the user time, the beam orbit is maintained at the reference orbit by an orbit feedback system. In the vertical direction, the feedback was performed at a high repetition rate as $10 \mathrm{~ms}$. In order to improve the stability and reliability of the beam position measurement, we have continued the beam based alignment of BPM. The remnant errors in the BPM offsets were estimated at all BPMs. A systematic error was found out at a specific part of the ring. We could fix a BPM that had an offset drift depending on the beam current. The amount of the drift was determined by the beam-based measurement.
\end{abstract}

\section{STABILIZATION OF BEAM ORBIT}

Photon Factory storage ring is the $2.5 \mathrm{GeV}$ synchrotron radiation source. It is a dedicated machine that has 28 of bending magnets in a circumference of $187 \mathrm{~m}$. There are several straight sections of 4 or $5 \mathrm{~m}$ in length. Five undulators (or multi-pole wigglers) and a superconducting wiggler were installed in those sections.

A big reconstruction for a high brilliant lattice was accomplished at four years ago. The beam emittance in the usual operation settled on about $35 \mathrm{~nm}$-rad. At the reconstruction, quadrupole magnets in a half part of the ring were replaced and the number of them was increased. The beam position monitors were also replaced attached to the new vacuum duct at the same section.

Beam lifetime of the PF ring is over 50 hours at a typical current of $450 \mathrm{~mA}$. 24-hour user run is provided with a single injection a day. After the injection at every morning, the COD is corrected to the reference orbit. During the user time, the COD is continuously measured at a high repetition rate, about 100 times a second. On the console of the BPM controller, the COD is updated every second. The resolution of the BPM was an order of 1 micrometer at that period. In the vertical direction, an orbit feedback loop operates at the same repetition rate as the COD measurement. There are 28 steering magnets for the feedback use. Those steering magnets are directly controlled by the same VME computer as one controls the BPM system. The feedback loop is closed using a digital signal processor contained in the VME. Usually variable factors of the feedback are set with stability as the highest priority. The feedback has a moderate frequency response up to $0.5 \mathrm{~Hz}$. In the horizontal direction, the orbit feedback is performed as an RF frequency feedback.
Without the vertical orbit feedback, distortion of the orbit would be about $0.2 \mathrm{~mm}$ in amplitude at the end of a 24-hour user run. The distortion was suppressed within about 20 micrometers or 30 micrometers at the most part of the ring. But at the specific part, the distortion grew up to over 50 micrometers. We wanted to know how the stability of the BPM was related to the orbit distortion, or whether there is any drift in the BPM offset at the specific region. It is difficult to detect a small drift of the offset by a method other than the beam-based measurement.

We prepared a beam-based alignment system using an electronic load. In the following sections, we will report on the beam-based measurement.

\section{BEAM BASED ALIGNMENT OF BPM}

\subsection{Measurement Method}

We have a quadrupole magnet switch system that connects an electronic load to a selected magnet. It was originally prepared for a beta function measurement. A scheme of the system is shown in figure 1.

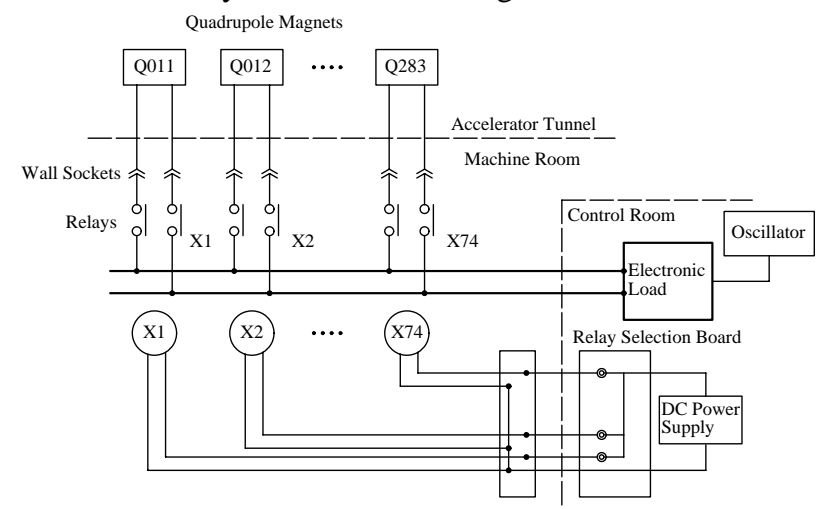

Figure 1: Electronic load switch system for the quadrupole magnets.

For the beam-based alignment, we controlled the electronic load by using an external oscillator. The current of a quadrupole magnet was altered by an amplitude of $3 \mathrm{~A}$ and by a frequency at $5 \mathrm{~Hz}$ typically. In this condition, the orbit oscillation at a few micrometers in amplitude was observed. The orbit oscillation was measured by a BPM at another portion of the ring. The beam-position output of the BPM is connedted to a FFT analyzer. The $5-\mathrm{Hz}$ component of the oscillation could be detected by the FFT analyzer with enough stability and sensitivity. We tried to use a lock-in amplifier to avoid a disturbance by a mechanical oscillation of the BPM itself, 
but the performance was not so good as expected. We preferred the FFT analyzer.

A local bump was prepared for the pair of the specific quadrupole magnet and the nearest BPM. When the amplitude of the orbit oscillation was plotted as a function of the BPM readings, we could obtain a graph as figure 2 . We could draw two straight lines that linked the data points. Two lines crossed near the bottom axis. The slopes of the both lines were symmetric about the intersection. The intersection was assumed to be an orbit at the center of the magnetic field. The reading of the BPM at the intersection was the BPM offset to the magnetic center. In the figure 2 , the local bump was created in the vertical direction. BPM offset in vertical and horizontal direction could be measured separately each other.

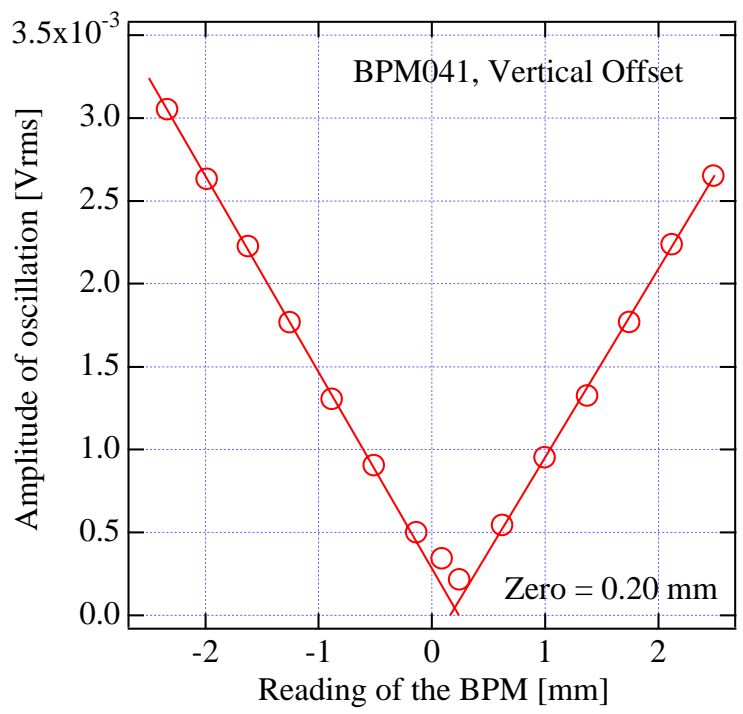

Figure 2: Measurement of the BPM offset.

\subsection{Distribution of BPM offsets around the ring}

In figure 3, vertical offset data for the all BPMs are plotted. As mentioned above, at the reconstruction of high brilliant lattice, we replaced vacuum duct in the half portion of the ring. In that part, we adopted 4-electrode BPM. In the other part of the ring, 6-elctrode BPM was installed since the first commissioning. Total number of BPM was 65. Among them, 42 BPMs were newly installed 4-elctrlode BPMs. In the figure 3, the BPMs at the range from No.7 to No.27 and from No.40 to No.60 are 4-elctrode type. For all BPMs, calibration on the electrical center was performed at the bench before the installation. The nominal tolerance about the mechanical alignment was a few hundred micrometers.

Most offsets of the BPM were distributed within +-0.5 $\mathrm{mm}$. But there are some portions where the large offset values were concentrated. In the central part of the graph, there is a remarkable hill over $1 \mathrm{~mm}$. It means that a systematic error is predominant among the 6-electrode BPMs. Such a situation turns out in the histogram for the 6-electrode BPM in figure 4 . The center of the distribution obviously shifts to the positive offset. We think it is worth correcting the systematic errors, because such large errors are observed at the insertion devices' sections. In the histogram for the 4-electrode BPM, the offsets are distributed almost symmetrically around zero. The standard deviation is $0.47 \mathrm{~mm}$. But there are several BPMs that have large offset values of about $1 \mathrm{~mm}$. We have to investigate what is the origin of such large error.

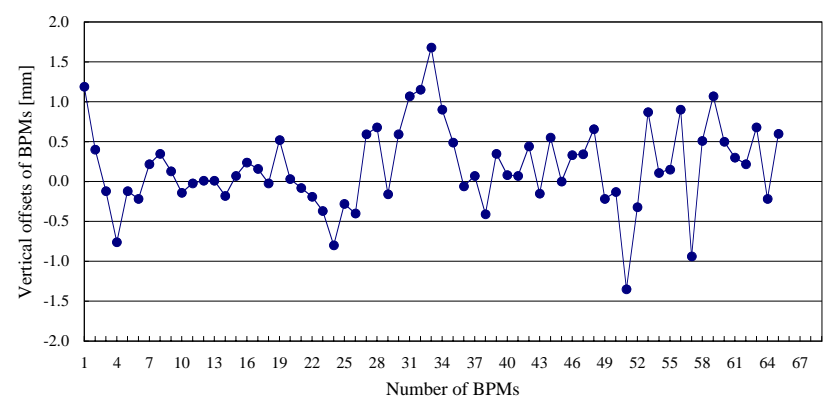

Figure 3: Vertical offsets of the all BPMs.
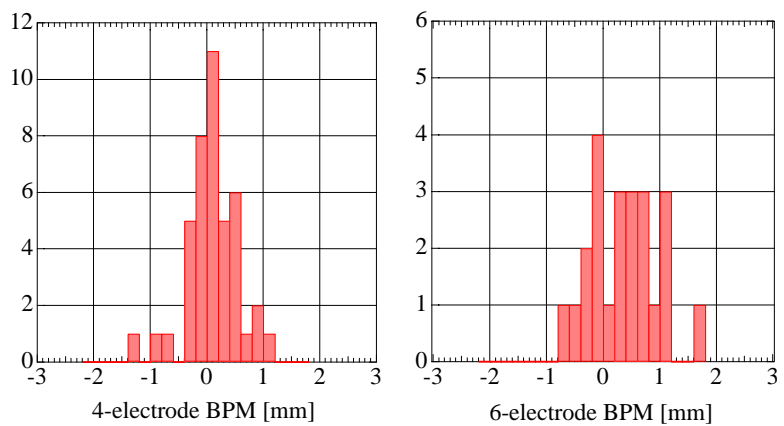

Figure 4: Histogram of the BPM offsets. left: 4-electrode BPMs, right: 6-electrode BPMs.

What was the accuracy of the beam-based measurement? At a specific BPM, No.65 in the figure 3, we measured the offset seven times during about one year from the preliminary stage of our beam-based alignment. The statistics of the data was summarized as $0.58 \mathrm{~mm}+$ $0.04 \mathrm{~mm}$. When the offset is detected from a measurement of a good signal-to-noise ratio such as the figure 2, the accuracy of the measurement is expected to be as good as the BPM-No.65. But it must be mentioned that, not all BPM offsets could be detected by such a good signal-to-noise measurement. At some BPMs, the two straight lines in the amplitude data intersected at far above the bottom axis. The orbit oscillation was not damped to zero around the intersection point. It may be a result of a coupling between the vertical and horizontal directions. The most troublesome situation was that the symmetry of the two straight lines was not hold. The origin of this phenomenon was not yet assigned. And such situation was frequently appeared with a large offset value. We have to take care the reliability of such data.

When there is any slope in the original orbit, the measured offsets will be displaced from the true value. We practically measured this effect by made an extra 
slope by a single kick of a steering magnet. The measurement results were shown in figure 5. The local bump for the quadrupole magnet was the same as the previous measurement. Another steering magnet was excited in several strengths. The horizontal axes of the graphs in the figure 5 are the calculated slopes. BPM013 was a 6-electrode type, and it was separated from the quadrupole by $0.26 \mathrm{~m}$. BPM211 is a 4-electrode BPM, and was separated from the quadrupole by $0.05 \mathrm{~m}$. The measured displacements for both BPMs were 0.63 $\mathrm{mm} / \mathrm{mrad}$ and $0.23 \mathrm{~mm} / \mathrm{mrad}$, respectively. Those values were two or four times larger than the simple estimations from the angles and distances. The orbit slopes estimated by the linear calculation may be overestimated.
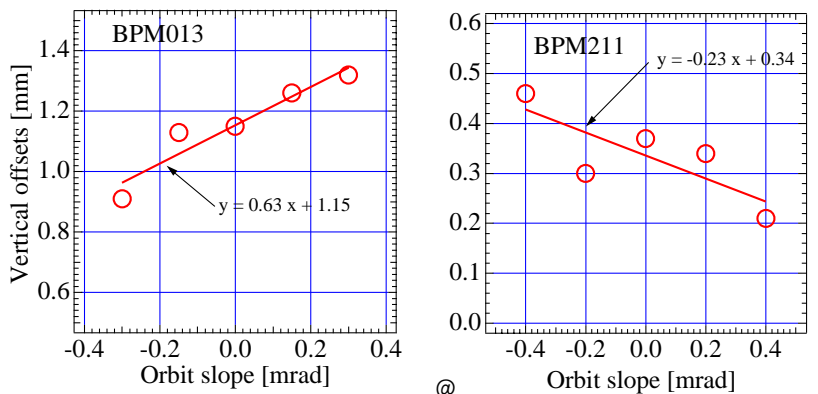

Figure 5: Effect of orbit slope made by a steering magnet.

A single kick of 0.3 mrad makes a COD of which amplitude is about $2 \mathrm{~mm}$. On the other hand, amplitude of COD appeared in the reference orbit or the usual orbit of the PF ring is less than $1 \mathrm{~mm}$. Possible slope appeared in the usual orbit is as large as $0.1 \mathrm{mrad}$. When the BPM has the rate of $0.6 \mathrm{~mm} / \mathrm{mrad}$, slope of $0.1 \mathrm{mrad}$ displace the offset only by $0.06 \mathrm{~mm}$. It is clear that the observed offset as large as $1 \mathrm{~mm}$ is not a small value that is made by any actual orbit slope in the ordinary orbit.

\subsection{Drift of the BPM Offset as a Function of Beam Current}

By using the beam-based measurement method, offset drift was practically detected as a function of beam current.

At a single-bunch mode, vertical beam positions at a few BPMs could not be aligned to the reference orbit by a usual COD correction procedure. The BPM called by a code 283 is one of such BPMs. When rather high current was stored in the single bunch, some portions of the vacuum duct suffered from heating up. We also have a 3 $\mathrm{GeV}$ mode operation during a week every six months. In the $3-\mathrm{GeV}$ mode, the vertical position at the BPM283 protruded from the reference orbit line. So we speculated that a few BPMs including the BPM283 had the offset drift depending on the temperature change.

In figure 6, the measurement result on the BPM283 is shown with the data on BPM281. The both BPM are the same type, and installed at both sides of a triplet quadrupoles. The horizontal axis is the stored beam current in the single bunch mode. It is clear that the BPM283 did drift as large as $0.3 \mathrm{~mm}$ under the high current. We are going to investigate the mechanical drift of the BPM283 and the temperature of the button electrodes themselves.

On the other hand, it is also clear that the BPM281 did not suffer from any drift. Beam positions at the most BPMs could be aligned to the reference orbit by the COD correction. We think a number of the BPMs that suffer from the offset drift is not so large. We will be able to make clear which BPM has offset drift by using the beam-based measurement.

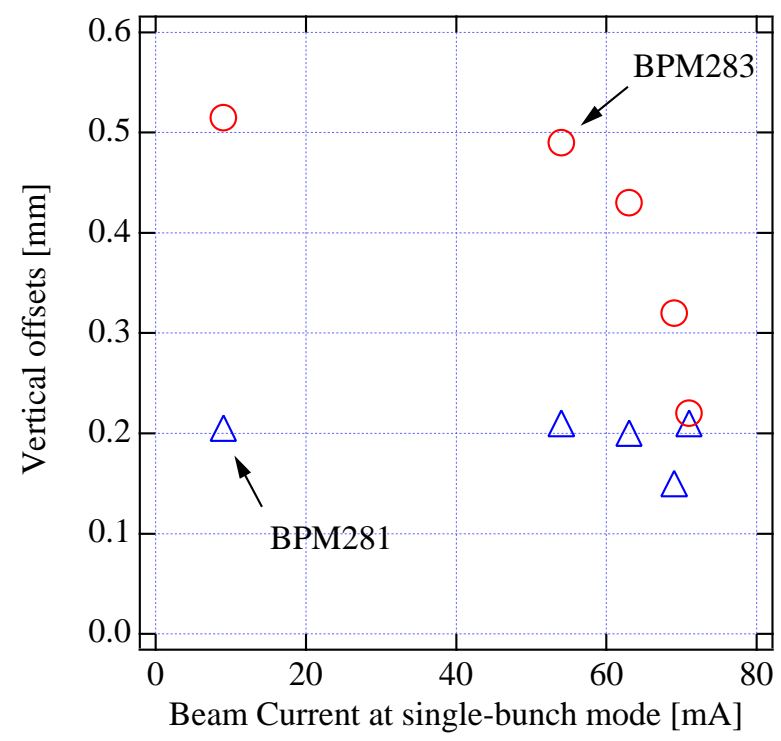

Figure 6: Variation of vertical offset as a function of beam current was observed at a specific BPM.

\section{CONCLUSION}

The beam-based alignment method using the electronic load was accomplished. Vertical offsets of all the BPM were measured and the distribution of the offsets were studied. Systematic errors were found out at the 6electrode BPM. Such error was observed at the region of the insertion devices. We are going to try the correction of the systematic error.

Using the beam-based method, we could measure the offset drift as a function of beam current. It turned out that not all BPM suffered from the drift.

A global alignment of quadrupoles is performed at several years. We would like to speculate the shape of actual COD using the last data of the mechanical alignment integrated with the beam-based alignment. 